Revista Brasileira de Agricultura Irrigada v.12, nº.3, p. 2692 - 2701, 2018

ISSN 1982-7679 (On-line)

Fortaleza, CE, INOVAGRI - http://www.inovagri.org.br

DOI: $10.7127 /$ rbai.v12n300869

Protocolo 869.18 - 12/01/2018 Aprovado em 22/05/2018

\title{
CALIBRAÇÃO DA EQUAÇÃO DE HARGREAVES-SAMANI PARA ESTIMAR A EVAPOTRANSPIRAÇÃO DE REFERÊNCIA NO ESTADO DO ESPÍRITO SANTO
}

\author{
Sidney Sára Zanetti ${ }^{1}$, Rafael Esteves Dohler ${ }^{2}$, Emerson Bastos do Carmo ${ }^{3}$, Roberto Avelino \\ Cecílio $^{4}$
}

\begin{abstract}
RESUMO
A maioria das localidades brasileiras não possui dados meteorológicos disponíveis para estimar a evapotranspiração de referência (ETo) pelo método padrão de Penman-Monteith (PM). Com isso, tornam-se importantes os métodos que possibilitam estimar a $\mathrm{ET}_{0}$ utilizando dados limitados. O presente estudo consistiu em calibrar a equação de Hargreaves-Samani (HS) por diferentes métodos para estimar a ET 0 diária no estado do Espírito Santo. Foram utilizados dados meteorológicos de nove estações automáticas do INMET localizadas no estado do Espírito Santo, sendo a ETo calculada pelo método de PM utilizada como referência para a calibração da equação de HS. A calibração foi realizada utilizando-se os dados de cada estação meteorológica individualmente, por três diferentes métodos: ajuste somente do parâmetro $\mathrm{H}_{\mathrm{c}}$ da equação de HS; ajuste dos três parâmetros simultaneamente $\left(\mathrm{H}_{\mathrm{c}}, \mathrm{H}_{\mathrm{e}} \mathrm{e} \mathrm{H}_{\mathrm{t}}\right)$; e ajuste da ETo utilizando regressão linear simples. As equações ajustadas obtidas foram avaliadas através do coeficiente de determinação $\left(\mathrm{r}^{2}\right)$ da regressão linear simples e a raiz do quadrado médio do erro (RQME). Embora a calibração por ajuste simultâneo dos três parâmetros da equação de HS tenha proporcionado melhores resultados, sua eficiência não diferiu expressivamente dos outros métodos. Dessa forma, considerando as limitações desse método, conclui-se que a calibração usando regressão linear seja o método mais indicado. Como segunda opção, considera-se a calibração usando o ajuste somente do parâmetro $\mathrm{H}_{\mathrm{c}}$ da equação de Hargreaves-Samani.
\end{abstract}

Palavras-chave: dados limitados, Penman-Monteith, ajuste empírico de modelos.

\section{CALIBRATION OF HARGREAVES-SAMANI'S EQUATION TO ESTIMATE EVAPOTRANSPIRATION OF REFERENCE IN THE STATE OF ESPÍRITO SANTO}

\footnotetext{
${ }^{1}$ Engenheiro Agrônomo, D.Sc em Produção Vegetal, Professor Adjunto, Departamento de Ciências Florestais e da Madeira, Universidade Federal do Espírito Santo, Jerônimo Monteiro - ES, email: sszanetti@ yahoo.com.br.

${ }^{2}$ Engenheiro Florestal, Doutorando em Produção Vegetal, Universidade Federal do Espírito Santo, Alegre-ES, email: rafaelesteves_123@hotmail.com (autor correspondente).

${ }^{3}$ Graduando em Engenharia Florestal, Universidade Federal do Espírito Santo, Campus Alegre, Alto Universitário, s/no, Guararema, Alegre - ES, CEP 29500-000, e-mail: bastosemerson787@gmail.com.

${ }^{4}$ Engenheiro Agrícola, D.Sc em Eng. Agrícola, Professor Associado, Departamento de Ciências Florestais e da Madeira, Universidade Federal do Espírito Santo, Jerônimo Monteiro - ES, email: racecilio@ yahoo.com.br.
} 


\begin{abstract}
Most Brazilian localities do not have available meteorological data to estimate reference evapotranspiration (ETo) by the standard Penman-Monteith (PM) method. Thus, methods that allow estimation of ETo using limited data become important. The present study consisted in calibrating the Hargreaves-Samani equation (HS) by different methods to estimate daily $\mathrm{ET}_{0}$ in the state of Espírito Santo. Meteorological data from nine INMET automatic stations located in the state of Espírito Santo were used, being ET 0 calculated by the PM method used as reference for the calibration of the HS equation. The calibration was performed using the data from each meteorological station individually, by three different methods: adjust only the parameter $\mathrm{H}_{\mathrm{c}}$ of the HS equation; adjustment of the three parameters simultaneously $\left(\mathrm{H}_{\mathrm{c}}, \mathrm{H}_{\mathrm{e}}\right.$ and $\left.\mathrm{H}_{\mathrm{t}}\right)$; and ETo adjustment using simple linear regression. The adjusted equations obtained were evaluated through the coefficient of determination $\left(\mathrm{r}^{2}\right)$ of the simple linear regression and the root mean square error (RQME). Although calibration by simultaneous adjustment of the three parameters of the HS equation gave better results, its efficiency did not differ significantly from the other methods. Thus, considering the limitations of this method, it is concluded that the calibration using linear regression is the most indicated method. As a second option, calibration using the adjustment of the $\mathrm{H}_{\mathrm{c}}$ parameter of the Hargreaves-Samani equation is considered.
\end{abstract}

Keywords: limited data, Penman-Monteith, empirical modeling

\section{INTRODUÇÃO}

Evapotranspiração (ET) é definida como a combinação das perdas de água por evaporação (de corpos hídricos, do solo e da superfície das plantas) e por transpiração dos cultivos. Sua determinação correta é importante para aplicações em diversas áreas, como hidrologia, agrometeorologia, irrigação, entre outros. Porém, a sua mensuração direta é difícil e onerosa, requerendo equipamentos e pessoal especializados. Por esse motivo, e por ter custos relativamente menores, maior simplicidade e praticidade, a utilização de métodos indiretos para estimar a evapotranspiração é frequentemente preferida em aplicações relacionadas ao manejo da irrigação (ALENCAR et al., 2015; PEREIRA et al., 2015).

De acordo com Pereira et al. (2002), a escolha de um método para se estimar a evapotranspiração depende de uma série de fatores, sendo um deles a disponibilidade de dados meteorológicos. Quando existem dados disponíveis recomenda-se a utilização do método de Penman-Monteith-FAO (ALLEN et al., 1998) como padrão internacional para o cálculo da evapotranspiração de referência $\left(\mathrm{ET}_{0}\right)$.
Todavia, a utilização desse método necessita da medição de vários dados meteorológicos, os quais muitas vezes não estão disponíveis e, ou não são confiáveis, como é o caso da velocidade do vento e a radiação solar (MARTí et al., 2015). Desta forma, tornam-se importantes estudos de métodos que possibilitem estimar a $\mathrm{ET}_{0}$ utilizando o mínimo possível de variáveis meteorológicas, em especial, aquelas de fácil e confiável mensuração (SENTELHAS et al., 2010).

Quando os dados meteorológicos disponíveis são limitados, (ALLEN et al., 1998) recomendam que a $\mathrm{ET}_{0}$ seja estimada utilizando a equação de Hargreaves-Samani (HS), a qual depende somente da radiação solar extraterreste e da temperatura do ar (HARGREAVES; SAMANI, 1985). No entanto, vários estudos têm mostrado que o método HS superestima a $\mathrm{ET}_{0}$ em regiões úmidas e subestima em regiões secas (BEZERRA et al., 2014; CARVALHO et al., 2015; FANAYA JÚNIOR et al., 2012; KISI, 2014; TAGLIAFERRE et al., 2010). Assim, faz-se necessário que, para aplicação em outras regiões, a equação de HS deva ser calibrada, por meio do ajuste de seus parâmetros empíricos (ALLEN et al., 1998). 
A maioria das localidades brasileiras não possuem dados meteorológicos disponíveis para estimar a evapotranspiração pelo método de Penman-Monteith (FAO-56), como é o caso do estado do Espírito Santo. Como já se tem conhecimento que a equação de HS tende a superestimar a $\mathrm{ET}_{0}$ na região Sudeste do Brasil, são necessários estudos que viabilizam a aplicação da equação de HS, como por exemplo, os estudos realizados em Goiás (FERNANDES et al., 2012), São Paulo (CONCEIÇÃO, 2013) e Pernambuco (LIMA et al., 2013), além de estudos realizados em outros países (AGUILAR; POLO, 2011; GHAMARNIA et al., 2011; SENTELHAS et al., 2010; SPERNA WEILAND et al., 2012;
TRAJKOVIC, 2007).

Do exposto, o objetivo deste trabalho foi calibrar a equação de Hargreaves-Samani para estimar a evapotranspiração de referência diária no estado do Espírito Santo, utilizandose de diferentes métodos de calibração.

\section{MATERIAIS E MÉTODOS}

A região de estudo foi o estado do Espírito Santo, localizado entre os meridianos $39^{\circ} 38^{\prime}$ e $41^{\circ} 50^{\prime}$ de longitude oeste e os paralelos $17^{\circ} 52^{\prime}$ e $21^{\circ} 19^{\prime}$ de latitude sul, que ocupa uma área aproximada de $46.184 \mathrm{~km}^{2}$ (Figura 1).

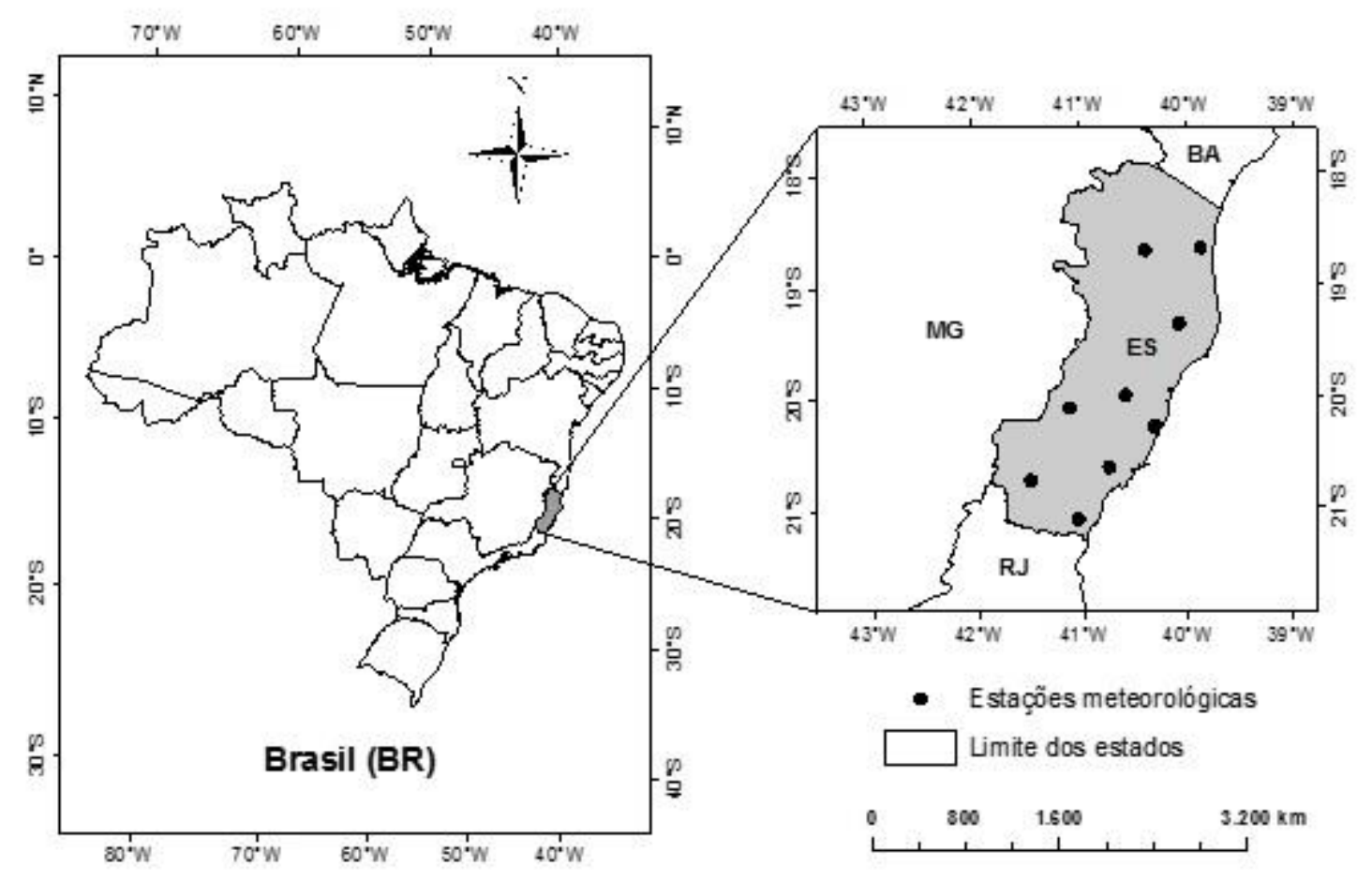

Figura 1. Localização da área em estudo, estado do Espírito Santo (ES), Brasil.

Segundo Alvares et al. (2013), são encontrados no estado do Espírito Santo os climas Af, Am e Aw, Cwc, Cfa, Cfb, Cwa, Cwb, com temperatura média anual entre 16,8 e $24{ }^{\circ} \mathrm{C}$; e pluviosidade média entre 1.078 e
$1.376 \mathrm{~mm}$ anuais. O presente estudo utilizou os dados meteorológicos diários das nove estações meteorológicas automáticas do INMET localizadas no estado do Espírito Santo (Tabela 1). 
Tabela 1. Estações meteorológicas automáticas do INMET utilizadas no estudo.

\begin{tabular}{|c|c|c|c|c|c|c|c|}
\hline Código & Nome & Estado & Latitude & Longitude & Altitude (m) & Período & $N^{0}$ de anos \\
\hline A612 & Vitória & ES & $-20^{\circ} 16^{\prime}$ & $-40^{\circ} 18^{\prime}$ & 9 & $2006 / 2015$ & 9 \\
\hline A613 & Santa Teresa & ES & $-19^{\circ} 59^{\prime}$ & $-40^{\circ} 34^{\prime}$ & 988 & $2007 / 2015$ & 8 \\
\hline A614 & Linhares & ES & $-19^{\circ} 21^{\prime}$ & $-40^{\circ} 04^{\prime}$ & 40 & $2006 / 2015$ & 9 \\
\hline A615 & Alfredo Chaves & ES & $-20^{\circ} 38^{\prime}$ & $-40^{\circ} 44^{\prime}$ & 35 & $2006 / 2015$ & 9 \\
\hline A616 & São Mateus & ES & $-18^{\circ} 42^{\prime}$ & $-39^{\circ} 50^{\prime}$ & 39 & $2006 / 2015$ & 9 \\
\hline A617 & Alegre & ES & $-20^{\circ} 45^{\prime}$ & $-41^{\circ} 29^{\prime}$ & 138 & $2006 / 2015$ & 9 \\
\hline A622 & $\begin{array}{l}\text { Presidente } \\
\text { Kennedy }\end{array}$ & ES & $-21^{\circ} 06^{\prime}$ & $-41^{\circ} 02^{\prime}$ & 80 & $2008 / 2015$ & 7 \\
\hline A623 & Nova Venécia & ES & $-18^{\circ} 41^{\prime}$ & $-40^{\circ} 23^{\prime}$ & 154 & $2008 / 2015$ & 7 \\
\hline A657 & Afonso Cláudio & $\mathrm{ES}$ & $-20^{\circ} 06^{\prime}$ & $-41^{\circ} 06^{\prime}$ & 520 & $2011 / 2015$ & 4 \\
\hline
\end{tabular}

Para cada estação foram obtidos os valores diários de todos os elementos meteorológicos necessários para calcular a evapotranspiração pelo método padrão de Penman-Monteith FAO-56 (ETOPM) conforme Equação 1 (ALLEN et al., 1998).

$$
E T_{0_{P M}}=\frac{0,408 \Delta(R n-G)+\gamma \frac{900}{(T+273)} U_{2}(e s-e a)}{\Delta+\gamma\left(1+0,34 U_{2}\right)}
$$

Em que:

$\mathrm{ET}_{0 \mathrm{PM}}=$ evapotranspiração de referência calculada pelo método de Penman-Monteith FAO-56 ( $\left.\mathrm{mm} \mathrm{dia}^{-1}\right) ; \Delta=$ inclinação da curva de saturação do vapor da água $\left(\mathrm{kPa}^{\circ} \mathrm{C}^{-1}\right) ; \mathrm{Rn}=$ radiação solar líquida $\left(\mathrm{MJ} \mathrm{m}^{-2} \mathrm{dia}^{-1}\right) ; \mathrm{G}=$ fluxo de calor no solo $\left(\mathrm{MJ} \mathrm{m}^{-2} \mathrm{dia}^{-1}\right) ; \gamma=$ constante psicrométrica $\left(\mathrm{kPa} \quad{ }^{\circ} \mathrm{C}^{-1}\right) ; \mathrm{U}_{2}=$ velocidade média do vento a $2 \mathrm{~m}$ de altura $\left(\mathrm{m} \mathrm{s}^{-}\right.$ ${ }^{1}$ ); es = pressão de saturação do vapor d'água no ar $(\mathrm{kPa})$; ea = pressão real do vapor d'água no ar $(\mathrm{kPa})$.
As variáveis $\Delta, \mathrm{Rn}, \gamma$, es e ea foram calculadas conforme (ALLEN et al., 1998) . Considerou-se o valor de $\mathrm{G}$ igual a zero e o albedo da cultura de referência igual a 0,23 . Os dias que apresentaram falhas em qualquer variável foram descartados da análise.

A $\mathrm{ET}_{0}$ calculada pelo método de Penman-Monteith FAO-56 foi utilizada como referência para a calibração da equação de Hargreaves-Samani SAMANI, 1985) - Equação 2.

$$
E T_{0 H S}=0,408 H_{c} R a\left(T_{\text {max }}-T_{\text {min }}\right)^{H e}\left(T_{\text {med }}+H_{t}\right)
$$

Em que, ET0Hs: evapotranspiração de referência obtida pelo método de HargreavesSamani $\left(\mathrm{mm} \mathrm{dia}{ }^{-1}\right) ; \mathrm{T}_{\max }, \mathrm{T}_{\min }$ e $\mathrm{T}_{\text {med: }}$ : temperatura máxima, mínima e média diária do ar $\left({ }^{\circ} \mathrm{C}\right)$, respectivamente; Ra: radiação solar extraterrestre $\left(\mathrm{MJ} \mathrm{m}^{-2} \mathrm{dia}^{-1}\right) ; \mathrm{H}_{\mathrm{c}}, \mathrm{H}_{\mathrm{e}}$ e $\mathrm{H}_{\mathrm{t}}$ : parâmetros da equação de Hargreaves-Samani.
Os parâmetros $\mathrm{H}_{\mathrm{c}}, \mathrm{H}_{\mathrm{e}}$ e $\mathrm{H}_{\mathrm{t}}$, na equação original de Hargreaves-Samani, equivalem a $0,0023, \quad 0,5$ e 17,8 , respectivamente (HARGREAVES; ALLEN, 2003). A radiação solar extraterrestre (Ra) foi calculada utilizando as equações recomendadas por (ALLEN et al., 1998). A temperatura média 
diária do ar $\left(\mathrm{T}_{\mathrm{med}}\right)$ foi obtida pela média aritmética entre as temperaturas máxima e mínima diárias do ar $\left(\mathrm{T}_{\min } \mathrm{e} \mathrm{T}_{\max }\right)$.

A equação de Hargreaves-Samani foi calibrada por meio do ajuste de seus parâmetros empíricos utilizando três diferentes métodos: a) ajuste somente do parâmetro $\mathrm{H}_{\mathrm{c}}$, mantendo-se os outros dois parâmetros $\left(\mathrm{H}_{\mathrm{e}} \mathrm{e}\right.$ $\mathrm{H}_{\mathrm{t}}$ ) fixos com seus valores originais; b) ajuste de todos os três parâmetros simultaneamente $\left(\mathrm{H}_{\mathrm{c}}, \mathrm{H}_{\mathrm{e}} \mathrm{e} \mathrm{H}_{\mathrm{t}}\right)$; e c) ajuste da $\mathrm{ET}_{0}$ utilizando regressão linear simples, conforme recomendado no boletim FAO-56 (ALLEN et al., 1998).

O ajuste por regressão linear simples foi realizando utilizando a seguinte equação:

$$
\mathrm{ET}_{0}=\mathrm{a}+\mathrm{b} \mathrm{ET}_{0 \mathrm{HS}}
$$

Para ajustar a Equação 3, pelo método dos mínimos quadrados linear, adotou-se como variável dependente $\left(\mathrm{ET}_{0}\right)$ a evapotranspiração calculada pelo método Penman-Monteith FAO-56 (ЕT0РM), e como variável independente a evapotranspiração estimada pela equação original de Hargreaves-Samani. Assim, após o ajuste da equação, esta foi aplicada para a correção da evapotranspiração estimada pela equação original de HargreavesSamani, utilizando os parâmetros a e b, gerando uma evapotranspiração calibrada (corrigida).

Os parâmetros empíricos da equação de Hargreaves-Samani $\left(\mathrm{H}_{\mathrm{c}}, \mathrm{H}_{\mathrm{e}}\right.$ e $\left.\mathrm{H}_{\mathrm{t}}\right)$ foram ajustados utilizando a Equação 2 como modelo, e utilizando o comando $\mathrm{nls}$ (non linear leastsquares), do pacote MASS, do programa estatístico $\mathrm{R}$ (ajuste de regressão não-linear pelo método dos mínimos quadrados).

A calibração foi realizada individualmente para cada estação meteorológica. As equações ajustadas obtidas foram avaliadas comparando-se a evapotranspiração calibrada com a evapotranspiração calculada pelo método Penman-Monteith FAO-56 (ETOPM), usando o coeficiente de determinação $\left(r^{2}\right)$ da regressão linear simples e a raiz do quadrado médio do erro (RQME) - Equação 4. Os ajustes foram, também, avaliados a partir de gráficos de dispersão entre os dados observados e estimados.

$$
R Q M E=\sqrt{n^{-1} \sum_{i=1}^{n}\left(E_{i}-O_{i}\right)^{2}}
$$

Em que, n: número de observações; $E_{\mathrm{i}}$ : evapotranspiração estimada pela equação calibrada de Hargreaves-Samani (mm dia $\left.{ }^{-1}\right)$; e $\mathrm{O}_{\mathrm{i}}$ : evapotranspiração calculada pelo método Penman-Monteith FAO-56 (mm dia $\left.{ }^{-1}\right)$.

\section{RESULTADOS E DISCUSSÃO}

Na Tabela 2 são apresentados os valores dos parâmetros obtidos a partir dos ajustes dos métodos de calibração da equação de Hargreaves-Samani para as estações meteorológicas do INMET localizadas no estado do Espírito Santo.

Tabela 2. Resultados do ajuste dos métodos de calibração da equação de Hargreaves-Samani para as

\begin{tabular}{|c|c|c|c|c|c|c|c|c|c|c|}
\hline \multirow{2}{*}{ Código } & \multirow{2}{*}{ Nome } & \multicolumn{3}{|c|}{ Regressão linear } & \multirow{2}{*}{$\mathbf{H}_{\mathbf{c}}$} & \multirow{2}{*}{$\mathbf{r}^{2}$} & \multicolumn{4}{|c|}{ Ajuste de todos os parâmetros } \\
\hline & & $\mathbf{a}$ & b & $\mathbf{r}^{2}$ & & & $\mathbf{H}_{\mathbf{c}}$ & $\mathbf{H}_{\mathrm{e}}$ & $\mathbf{H}_{\mathrm{t}}$ & $\mathbf{r}^{2}$ \\
\hline \multicolumn{2}{|c|}{ A612 Vitória } & $-1,112$ & 1,236 & 0,81 & 0,0022 & 0,81 & 0,0024 & 0,710 & 0,00 & 0,83 \\
\hline \multicolumn{2}{|c|}{ A613 Santa Teresa } & $-0,100$ & 0,943 & 0,68 & 0,0021 & 0,68 & 0,0030 & 0,553 & 3,68 & 0,70 \\
\hline \multicolumn{2}{|c|}{ A614 Linhares } & $-0,423$ & 1,134 & 0,78 & 0,0024 & 0,78 & 0,0021 & 0,729 & 4,66 & 0,82 \\
\hline \multicolumn{2}{|c|}{ A615 Alfredo Chaves } & $-0,671$ & 1,082 & 0,80 & 0,0021 & 0,80 & 0,0014 & 0,812 & 5,74 & 0,85 \\
\hline \multicolumn{2}{|c|}{ A616 São Mateus } & $-0,710$ & 1,143 & 0,80 & 0,0022 & 0,80 & 0,0028 & 0,641 & 0,00 & 0,82 \\
\hline \multicolumn{2}{|c|}{ A617 Alegre } & $-0,261$ & 0,953 & 0,84 & 0,0020 & 0,84 & 0,0018 & 0,613 & 11,23 & 0,85 \\
\hline
\end{tabular}
estações meteorológicas do INMET localizadas no estado do Espírito Santo. 


\begin{tabular}{cccccccccc} 
A622 Pres. Kennedy & $-0,776$ & 1,341 & 0,70 & 0,0026 & 0,70 & 0,0040 & 0,560 & 0,00 & 0,72 \\
A623 Nova Venécia & $-0,185$ & 0,906 & 0,70 & 0,0020 & 0,70 & 0,0015 & 0,606 & 17,83 & 0,71 \\
A657 Afonso Cláudio & $-0,066$ & 0,914 & 0,70 & 0,0021 & 0,70 & 0,0012 & 0,585 & 37,05 & 0,71 \\
\hline Média & $-0,478$ & 1,072 & 0,76 & 0,0022 & 0,76 & 0,0022 & 0,645 & 8,91 & 0,78 \\
\hline
\end{tabular}

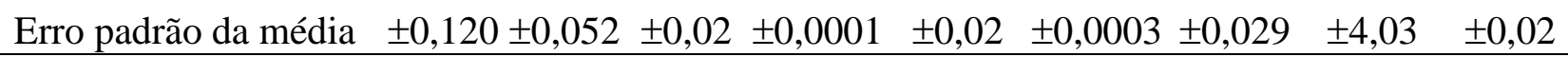

No ajuste por regressão linear, recomendado no boletim FAO-56 (ALLEN et al., 1998), os valores do parâmetro b (inclinação da reta) apresentaram valores próximos da unidade, com média igual a 1,072, e baixa variabilidade entre as estações, conforme indicado pelo reduzido valor do erro padrão da média $( \pm 0,052)$. Nesse tipo de análise, valores de inclinação da reta próximos do valor unitário indicam a existência de um erro aproximadamente sistemático na evapotranspiração estimada pelo método de HS, em relação à evapotranspiração estimada pelo método de Penman-Monteith, erro esse equivalente ao valor do intercepto da reta (parâmetro a). Podese observar que todos os valores de intercepto (a) foram negativos, com média de $-0,478 \mathrm{~mm} / \mathrm{d} \pm 0,120 \mathrm{~mm} / \mathrm{d}$, o que significa que, em média, o método de HS superestima a ETopm no estado do Espírito Santo, confirmando que a calibração é um procedimento necessário (ALENCAR; SEDIYAMA; MANTOVANI, 2015; ALLEN et al., 1998). O fato do método de HS superestimar a evapotranspiração em climas úmidos é um comportamento já conhecido, relatado por diversos autores (ALENCAR; SEDIYAMA; MANTOVANI, 2015; ALLEN et al., 1998; CARVALHO et al., 2015; CONCEIÇÃO, 2013; FERNANDES et al., 2012; TRAJKOVIC, 2007).

Pode-se observar ainda, na Tabela 2, que os parâmetros $\mathrm{H}_{\mathrm{c}}$ ajustados apresentaram pouca variação em relação ao valor original de Hargreaves-Samani $(0,0023)$, com valor médio de 0,0022 e baixo erro padrão da média $( \pm 0,0001)$. Outros autores, também ajustando somente o parâmetro $\mathrm{H}_{\mathrm{c}}$ da equação de $\mathrm{HS}$ no Brasil, obtiveram valores próximos: Fernandes et al. (2012) obteve um valor médio de 0,0014 em Goiás; Conceição (2013) obteve um valor médio de 0,0018 em São Paulo; e Borges e
Mendiondo (2007) propuseram valor de 0,0022 para os meses de setembro a abril, e de 0,0020 para maio a agosto também no estado de São Paulo. Essas variações são consideradas normais, pois dependem das características climáticas de cada local estudado.

Na calibração da equação de HS a partir do ajuste simultâneo de todos os seus parâmetros $\left(\mathrm{H}_{\mathrm{c}}, \mathrm{H}_{\mathrm{e}} \mathrm{e} \mathrm{H}_{\mathrm{t}}\right)$, os valores obtidos apresentaram maior variação, sobretudo o parâmetro $\mathrm{H}_{\mathrm{t}}$, com variação de 0,00 a 37,05. O fato desse tipo de ajuste ser um processo estritamente matemático, implica na obtenção de valores discrepantes dos parâmetros entre as estações $\left(\mathrm{H}_{\mathrm{c}}, \mathrm{H}_{\mathrm{e}}\right.$ e $\left.\mathrm{H}_{\mathrm{t}}\right)$, conforme consta na Tabela 2.

Outro aspecto interessante sobre os ajustes realizados refere-se ao comportamento dos coeficientes de determinação $\left(\mathrm{r}^{2}\right)$ resultantes, também apresentados na Tabela 2 . $\mathrm{Na}$ calibração da equação de HS usando regressão linear e usando o ajuste único do parâmetro $\mathrm{H}_{c}$, não houve alteração dos valores de $\mathrm{r}^{2}$ em relação ao método original de HS. Isso ocorre, pois, esses dois métodos corrigem a ETo sem alterar os valores de $r^{2}$, ou seja, não alteram a dispersão dos dados. Por outro lado, na calibração usando o ajuste simultâneo de todos os parâmetros, observa-se uma ligeira melhoria dos coeficientes de determinação.

Na Tabela 3 constam os valores de raiz do quadrado médio do erro (RQME) calculados usando os dados de $\mathrm{ET}_{0}$ estimados pela equação de HS original e calibrada pelos diferentes métodos, em relação à evapotranspiração calculada pelo método de Penman-Monteith. A calibração usando o ajuste simultâneo de todos os parâmetros apresentou o melhor desempenho, com RQME médio de $0,70 \mathrm{~mm} / \mathrm{d}$, seguida da calibração usando regressão linear, com RQME de 0,73 $\mathrm{mm} / \mathrm{d}$, e da calibração usando apenas o ajuste do parâmetro $\mathrm{H}_{\mathrm{c}}$, com RQME de $0,75 \mathrm{~mm} / \mathrm{d}$. 
Tabela 3. Raiz do quadrado médio do erro (RQME, $\mathrm{mm} \mathrm{d}^{-1}$ ) da evapotranspiração de referência calculada pela equação de Hargreaves-Samani (HS) original e calibrada utilizando diferentes métodos, em relação ao método de Penman-Monteith FAO-56.

\begin{tabular}{llcccc}
\hline \multirow{2}{*}{ Código } & \multirow{2}{*}{ Nome } & \multirow{2}{*}{$\mathbf{H S}$} & \multicolumn{3}{c}{ HS calibrado } \\
\cline { 4 - 6 } & & original & Reg. linear & $\mathbf{H}_{\mathbf{c}}$ & $\mathbf{H}_{\mathbf{c}}, \mathbf{H}_{\mathbf{e}}, \mathbf{H}_{\mathbf{t}}$ \\
\hline A612 & Vitória & 0,74 & 0,67 & 0,76 & 0,65 \\
A613 & Santa Teresa & 0,80 & 0,74 & 0,74 & 0,73 \\
A614 & Linhares & 0,70 & 0,68 & 0,69 & 0,62 \\
A615 & Alfredo Chaves & 0,83 & 0,76 & 0,80 & 0,66 \\
A616 & São Mateus & 0,61 & 0,57 & 0,60 & 0,55 \\
A617 & Alegre & 0,78 & 0,62 & 0,63 & 0,60 \\
A622 & Presidente Kennedy & 1,27 & 1,06 & 1,10 & 1,02 \\
A623 & Nova Venécia & 0,96 & 0,73 & 0,73 & 0,72 \\
A657 & Afonso Cláudio & 0,87 & 0,75 & 0,75 & 0,74 \\
\hline \multicolumn{2}{c}{ Média } & $0,84 \pm 0,06$ & $0,73 \pm 0,05$ & $0,75 \pm 0,05$ & $0,70 \pm 0,05$ \\
\hline
\end{tabular}

A partir dos resultados apresentados na Tabela 3, pode-se observar que os melhores resultados foram obtidos para a estação meteorológica de São Mateus, e os piores resultados obtidos para a estação de

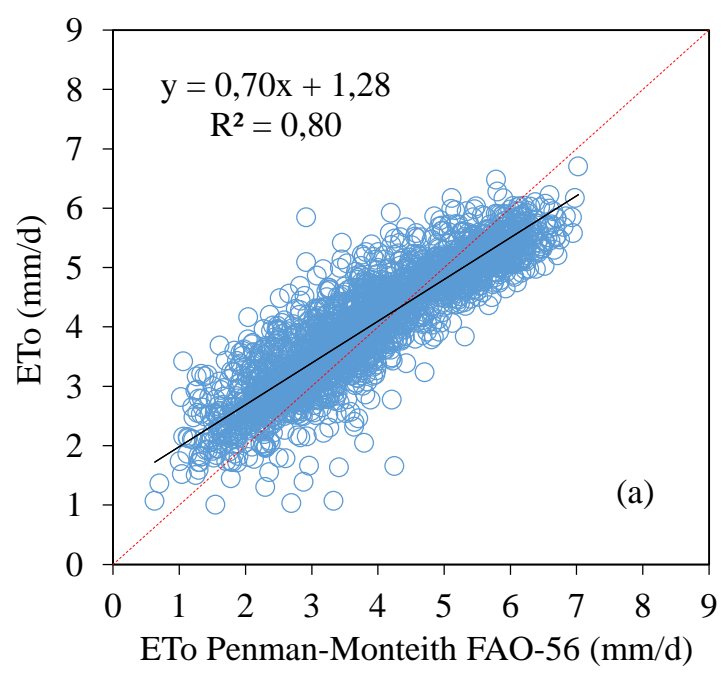

Presidente Kennedy. Nas Figuras 2 e 3 são apresentados os gráficos de dispersão referentes somente aos melhores e piores ajustes obtidos, para essas duas localidades.

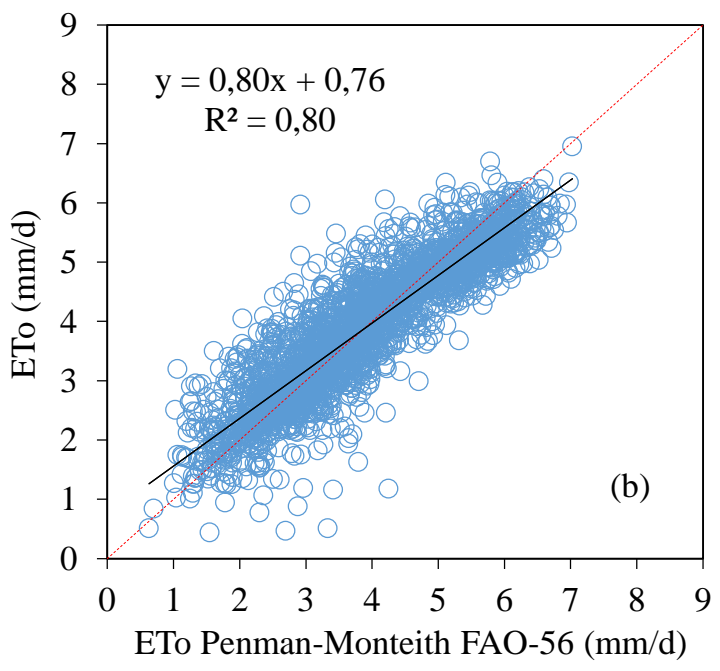



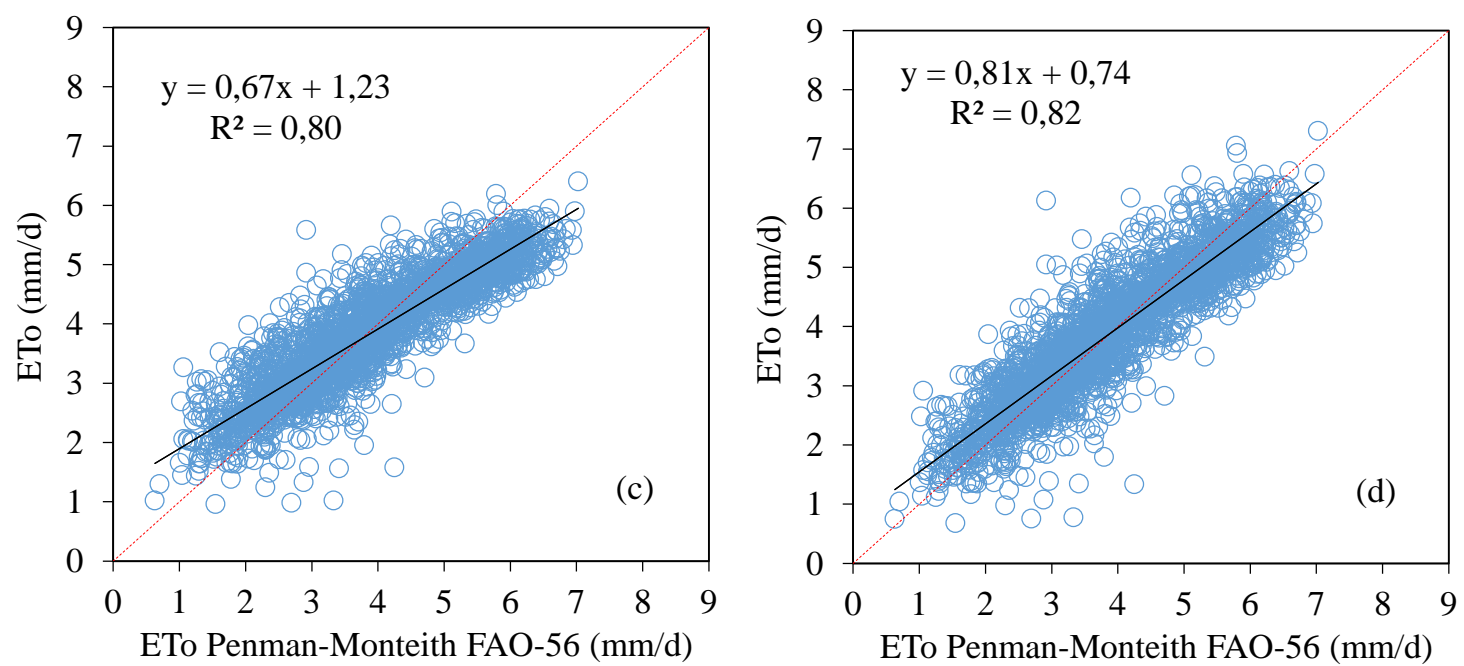

Figura 2. Dispersão da evapotranspiração de referência calculada pela equação de Hargreaves-Samani original (a), calibrada com regressão linear (b), com ajuste apenas de $\mathrm{H}_{\mathrm{c}}$ (c), e com ajuste de $\mathrm{H}_{\mathrm{c}}, \mathrm{H}_{\mathrm{e}}$ e $\mathrm{H}_{\mathrm{t}}(\mathrm{d})$, em relação ao método de Penman-Monteith FAO-56, para a estação meteorológica de São Mateus (melhores resultados).
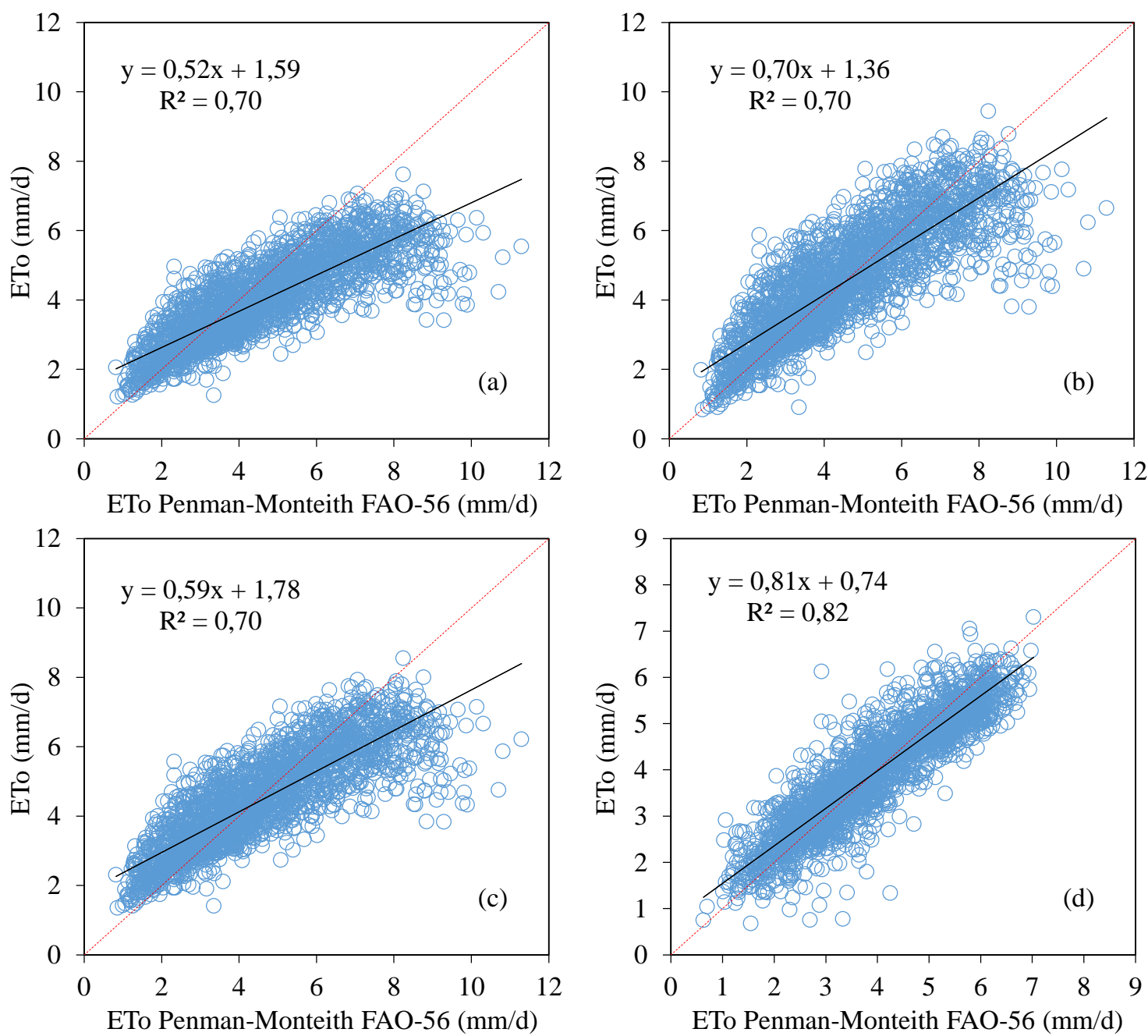

Figura 3. Dispersão da evapotranspiração de referência calculada pela equação de Hargreaves-Samani original (a), calibrada com regressão linear (b), com ajuste apenas de $\mathrm{H}_{\mathrm{c}}$ (c), e com ajuste de $\mathrm{H}_{\mathrm{c}}, \mathrm{H}_{\mathrm{e}}$ e $\mathrm{H}_{\mathrm{t}}(\mathrm{d})$, em relação ao método de Penman-Monteith FAO-56, para a estação meteorológica de Presidente Kennedy (piores resultados). 
Embora o método de calibração por ajuste simultâneo de todos os parâmetros da equação de HS tenha proporcionado melhores resultados (Tabela 3), sua eficiência não diferiu expressivamente dos demais métodos. Além disso, trata-se de um método mais complexo, que necessita de ajustes de regressão não-linear, e que apresenta parâmetros mais discrepantes entres as estações estudadas, sobretudo dos valores de $\mathrm{H}_{\mathrm{t}}$. Outra característica desse método, também supostamente incoerente, refere-se ao ajuste até mesmo do expoente da amplitude térmica da equação de $\mathrm{HS}\left(\mathrm{H}_{\mathrm{e}}\right)$, que na realidade se trata de uma raiz quadrada.

Dessa forma, devido à inexpressiva diferença apresentada entre os métodos de calibração testados, considera-se que o ajuste usando regressão linear (Equação 2) seja o método mais indicado, recomendado por (ALLEN et al., 1998). Trata-se de um método ainda pouco utilizado, sendo o estudo realizado por Lima et al. (2013) um exemplo desse tipo de aplicação. Como segunda opção, considerase a calibração a partir do ajuste somente do parâmetro $\mathrm{H}_{\mathrm{c}}$ da equação de HS, sendo este, segundo (MARTí et al., 2015), o método utilizado na maioria dos casos.

No presente estudo foram testados diferentes métodos de calibração da equação de HS a partir de ajustes locais, utilizando os dados meteorológicos de cada estação individualmente. Pelos resultados obtidos (Tabela 2) usando regressão linear e usando o ajuste único do parâmetro $\mathrm{H}_{\mathrm{c}}$, constatou-se a existência de certa similaridade nos resultados obtidos para as diversas localidades estudadas, indicada pelos baixos valores do erro padrão da média calculados. Esses resultados indicam a viabilidade de realização de futuros estudos visando a calibração regional da equação de HS para o estado do Espírito Santos, ou até mesmo para a região Sudeste do Brasil e, ou outras regiões, como por exemplo o trabalho realizados por Fernandes et al. (2012), no estado de Goiás, e outros estudos internacionais relevantes (MARTÍ et al., 2015; SAMANI, 2000; VANDERLINDEN et al., 2004).

\section{CONCLUSÃO}

A equação original de HargreavesSamani tende a superestimar a $\mathrm{ET}_{0}$ no estado do Espírito Santo, em relação ao método padrão de Penman-Monteith FAO-56.

Embora o método de calibração por ajuste simultâneo de todos os parâmetros da equação de Hargreaves-Samani tenha proporcionado melhores resultados, sua eficiência não diferiu expressivamente dos outros métodos testados. Dessa forma, considerando as limitações desse método, conclui-se que a calibração usando regressão linear seja o método mais indicado, embora pouco utilizado. Como segunda opção, considera-se a calibração usando o ajuste somente do parâmetro $\mathrm{H}_{\mathrm{c}}$ da equação de Hargreaves-Samani (método atualmente mais utilizado).

\section{REFERÊNCIAS BIBLIOGRÁFICAS}

AGUILAR, C.; POLO, M. J. Generating reference evapotranspiration surfaces from the Hargreaves equation at watershed scale. Hydrology and Earth System Sciences, v. 15, n. 8, p. 2495-2508, 2011.

ALENCAR, L. P. DE; SEDIYAMA, G. C.; MANTOVANI, E. C. Estimativa da evapotranspiração de referência (ETo padrão FAO), para Minas Gerais, na ausência de alguns dados climáticos. Engenharia Agrícola, v. 35, n. 1, p. 39-50, 2015.

ALLEN, R. G. et al. Crop evapotranpiration: guidelines for computing crop water requirements. Roma: FAO, 1998.

ALVARES, C. A. et al. Köppen's climate classification map for Brazil. Meteorologische Zeitschrift, v. 22, n. 6, p. 711-728, 1 dez. 2013.

BORGES, A. C.; MENDIONDO, E. M. Comparação entre equações empíricas para estimativa da evapotranspiração de referência na Bacia do Rio Jacupiranga. 
Revista Brasileira de Engenharia Agrícola e Ambiental, v. 11, n. 3, p. 293-300, 2007.

CARVALHO, D. F. DE et al. Estimativa da evapotranspiração de referência a partir de dados meteorológicos limitados. Pesquisa Agropecuária Brasileira, v. 50, n. 1, p. 1-11, 2015.

CONCEIÇÃO, M. A. F. Ajuste do modelo de Hargreaves para estimativa da evapotranspiração de referência no noroeste paulista. Revista Brasileira de Agricultura Irrigada, v. 7, n. 5, p. 306-316, 2013.

FERNANDES, D. S. et al. Calibração regional e local da equação de Hargreaves para estimativa da evapotranspiração de referência. Revista Ciência Agronômica, v. 43, n. 2, p. 246-255, 2012.

GHAMARNIA, $H$. et al. Time and Place Calibration of the Hargreaves Equation for Estimating Monthly Reference Evapotranspiration under Different Climatic Conditions. Journal of Agricultural Science, v. 4, n. 3, p. 111-122, 2011.

HARGREAVES, G. H.; ALLEN, R. G. History and Evaluation of Hargreaves Evapotranspiration Equation. Irrigation and Drainage Engineering, v. 129, n. 1, p. 53-63, 2003.

HARGREAVES, G. H.; SAMANI, Z. A. Reference crop evapotranspiration from ambient air temperature. 1985.
LIMA, J. R. S. et al. Calibration of HargreavesSamani equation for estimating reference evapotranspiration in sub-humid region of Brazil. Journal of water resources and protection, v. 5, p. 1-5, 2013.

MARTÍ, P. et al. Parametric expressions for the adjusted Hargreaves coefficient in Eastern Spain. Journal of Hydrology, v. 529, p. 17131724, out. 2015.

PEREIRA， A. R.; ANGELOCCI， L. R.; SENTELHAS, P. C. Agrometeorologia: Fundamentos e Aplicações Práticas. Lavras: Agropecuária, 2002.

SAMANI, Z. Estimating solar radiation and evapotranspiration using minimum climatological data. Journal of Irrigation and Drainage Engineering, v. 126, n. 4, p. 265267, 2000.

SPERNA WEILAND, F. C. et al. Selecting the optimal method to calculate daily global reference potential evaporation from CFSR reanalysis data for application in a hydrological model study. Hydrology and Earth System Sciences, v. 16, n. 3, 2012.

TRAJKOVIC, S. Hargreaves versus PenmanMonteith under Humid Conditions. Journal of Irrigation and Drainage Engineering, v. 133, n. 1, p. 38-42, 2007.

VANDERLINDEN, K.; GIRÁLDEZ, J. V.; VAN MEIRVENNE, M. Assessing Reference Evapotranspiration by the Hargreaves Method in Southern Spain. Journal of Irrigation and Drainage Engineering, v. 130, n. 3, p. 184 191, jun. 2004. 